\title{
Synthetic polyphosphate inhibits endogenous coagulation and platelet aggregation in vitro
}

\author{
XIAOYANG YANG ${ }^{1,2}$, MENGJIE WAN $^{1}$, TING LIANG $^{2}$, MINYUAN PENG $^{2}$ and FANGPING CHEN ${ }^{2,3}$ \\ ${ }^{1}$ Department of Hematology, Haikou Municipal Hospital, Affiliated Haikou Hospital of Central South University, Haikou, \\ Hainan 570208; ${ }^{2}$ Department of Hematology, Xiangya Hospital, Central South University, Changsha, Hunan 410008; \\ ${ }^{3}$ Department of Hematology, The Third Hospital of Xiangya Central South University, Changsha, Hunan 410013, P.R. China
}

Received April 11, 2016; Accepted July 27, 2016

DOI: $10.3892 /$ br.2016.816

\begin{abstract}
Platelet-derived polyphosphate has previously been indicated to induce coagulation. However, industrially synthesized polyphosphate has been found to have different effects from those of the platelet-derived form. The present study investigated whether synthetic sodium polyphosphate inhibits coagulation using routine coagulation tests and thromboelastography. Synthetic polyphosphate was found to inhibit adenosine diphosphate-, epinephrine-, arachidonic acid-, ristocetin-, thrombin-, oxytocin- and pituitrin-induced platelet aggregation. The effects of synthetic polyphosphate in clotting inhibition were revealed by the analysis of clotting factor activity and platelet aggregation tests. Synthetic polyphosphate may inhibit platelet aggregation by reducing platelet calcium levels, as indicated by the results of flow cytometric analysis and high-throughput fluorescent screening. Furthermore, analysis of thromboxane (TX) B2 by ELISA indicated that synthetic polyphosphate reduces platelet aggregation by inhibiting the TXA2 signaling pathway. In conclusion, synthetic polyphosphate inhibits clotting factor activity and endogenous coagulation by reducing the levels of calcium ions and TXA2 to curb platelet aggregation.
\end{abstract}

\section{Introduction}

Industrially synthesized polyphosphate (sodium polyphosphate) is a complex, glassy material of approximate

Correspondence to: Dr Xiaoyang Yang, Department of Hematology, Haikou Municipal Hospital, Affiliated Haikou Hospital of Central South University, 43 Renmin Road, Haikou, Hainan 570208, P.R. China

E-mail: y108108@126.com

Dr Fangping Chen, Department of Hematology, Xiangya Hospital, Central South University, 72 Xiangya Road, Changsha, Hunan 410008, P.R. China

E-mail: 128102109@csu.edu.cn

Key words: polyphosphate, routine coagulation test, platelet aggregation, thromboelastogram, high-throughput screening system composition $\left(\mathrm{NaPO}_{3}\right)_{\mathrm{x}}$, where $\mathrm{x} \geq 20$, but unknown constitution. It is produced by the fusion and rapid quenching of monosodium orthophosphate in accordance with the following reaction: $\mathrm{n}\left(\mathrm{NaH}_{2} \mathrm{PO}_{4}\right) \rightarrow\left(\mathrm{NaPO}_{3}\right)_{\mathrm{n}}+\mathrm{nH}_{2} \mathrm{O}(1,2)$. Polyphosphates are widely distributed in nature and are present in archaebacteria, eubacteria, fungi, algae and protozoa, as well as higher plants and animals $(3,4)$. However, as it has no known function, polyphosphate has previously been dismissed as a 'molecular fossil' (1). Recently, however, studies have indicated that long chain polyphosphate ( $>45$ phosphate units) may induce thrombosis and infection, linked with the activation of coagulation factor XII (FXII), via a bradykinin-dependent mechanism (5-7). However, Faxälv et al found that polyphosphate released from platelets did not activate FXII (5), and the relationship between synthetic polyphosphate, platelets and coagulation remains unclear. Therefore, the present study explored the effect of industrially synthesized polyphosphate on hemostasis and coagulation in a clinical laboratory setting and investigated the potential underlying mechanisms.

\section{Materials and methods}

Primary reagents and instruments. Synthetic polyphosphate (marketed as 'phosphate glass, water' or polyP), 4',6-diamidino2-phenylindole dihydrochloride (DAPI), Fluo-3 and adenosine diphosphate (ADP) were purchased from Sigma-Aldrich (Merck Millipore, Darmstadt, Germany). Other reagents were purchased from Aladdin (Shanghai, China) unless otherwise stated. To reduce the levels of very short polymers, the synthetic polyphosphate $(1 \mathrm{~g})$ was dissolved twice in $10 \mathrm{ml}$ purified water in a $10-\mathrm{ml}$ tube and then resuspended in $10 \mathrm{ml} 250 \mathrm{mM} \mathrm{LiCl}$ (6). The supernatant was considered as synthetic polyphosphate solution, and the residue was removed via centrifugation $(5,000 \mathrm{x}$ g for $10 \mathrm{~min})(6)$. The synthetic polyphosphate solution was stored at $4^{\circ} \mathrm{C}$.

Venous blood samples were collected by health worker volunteers. For each sample, $3 \mathrm{ml}$ blood was collected into a citrate anticoagulation tube, and platelet-poor plasma (PPP) was extracted within $30 \mathrm{~min}$. The blood was centrifuged at $1,000 \mathrm{x} \mathrm{g}$ for $10 \mathrm{~min}$, and the upper $1 \mathrm{ml}$, comprising the platelet-rich plasma (PRP), was collected. The PRP was centrifuged at $2,000 \mathrm{x} \mathrm{g}$ for $10 \mathrm{~min}$, and the upper $300 \mu \mathrm{l}$ was retained as PPP. All study participants gave informed, signed 
consent, and the study was approved by the Institutional Ethics Committee of Xiangya Hospital, Central South University (Changsha, China). The study was carried out in accordance with the Code of Ethics of the World Medical Association (Declaration of Helsinki) for experiments involving humans. The blood samples were collected in 1:9 sodium citrate human venous blood specimen collection containers for clinical testing (Shandong Weigao Group Medical Polymer Co., Ltd., Weihai, China). All of the products and instruments as well as the methods of platelet aggregation, coagulation routines, thromboelastograms, and clotting factor activity tests were appropriate for clinical assessment.

Evaluation of the effects of synthetic polyphosphate using routine coagulation tests. Different quantities $(0,5,10$, 20 and $40 \mu \mathrm{l})$ of synthetic polyphosphate in $\mathrm{LiCl}(250 \mathrm{mM})$ were added to $3 \mathrm{ml}$ whole blood, and a routine coagulation test was carried out. This test measured the prothrombin time (PT), the international normalized ratio (INR), fibrinogen level (FIB), the activated partial thromboplastin time (APTT), D-dimer (DD) level and the thrombin time (TT) using a high-throughput hemostasis analyzer (Destiny Max; Trinity Biotech Plc., Bray, Ireland). The assay kits were purchased from Beijing Biochem Medical Technology Co., Ltd. (Beijing, China). All analyses were based on disseminated intravascular coagulation (DIC) diagnostic criteria (8). All of the experiments were performed in triplicate.

Effect of synthetic polyphosphate on clotting factor activity. Different quantities $(0,10,20$ and $40 \mu \mathrm{l})$ of synthetic polyphosphate in $\mathrm{LiCl}(250 \mathrm{mM})$ were added to $3 \mathrm{ml}$ whole blood, and the activities of factor VIII (FVIII), factor IX (FIX), factor XI (FXI) and FXII were detected using Siemens Healthcare Diagnostics products (item ids: OTXW17, OTXX17, OSDF13 and OSDG13; Siemens Healthcare AG, Munich, Germany) in a high-throughput hemostasis analyzer. Coagulation factor-deficient plasma, provided with the diagnostic assay kits, was used to confirm factor activity and to identify and quantify the activity of coagulation factors in the treated plasma. A mixture of the respective factor-deficient plasma and the treated plasma was tested by APTT assay. All experiments were performed in triplicate.

Evaluation of the effects of synthetic polyphosphate using thromboelastography (TEG). Different quantities (20, 40 and $80 \mu \mathrm{l}$ ) of polyphosphate were added to $3 \mathrm{ml}$ whole blood in a citrated tube, and all TEG analyses were performed with a kaolin and $\mathrm{CaCl}_{2}$ active assay kit and TEG instrument (CFMS ${ }^{\mathrm{TM}}$; Lepu Medical Technology Co., Ltd., Beijing, China) (9). All experiments were performed in triplicate, and $250 \mathrm{mM} \mathrm{LiCl}$ was used as a control treatment. The following variables were determined: Reaction time (R); time taken to reach $20 \mathrm{~mm}$ amplitude $(\mathrm{K})$; angle degree; maximum amplitude (MA); shear elastic simulation strength $(\mathrm{G})$; time to maximum amplitude (TMA); coagulation index (CI); lysis time estimate (LTE); estimated percent lysis (EPL); and percentage of clot lysed after $30 \mathrm{~min}$ (LY30).

Effect of synthetic polyphosphate on platelet aggregation. Aggregation tests were performed via the turbidity method using PRP with a TYXN-96 multi-functional intelligent blood condensate meter (Shanghai General Electromechanical Technology Research Institute, Shanghai, China). A total of $200 \mu \mathrm{l}$ PRP was tested for aggregation. A $10 \mu \mathrm{l}$ aliquot of $250 \mathrm{mM} \mathrm{LiCl}$ with one of several platelet agonists was used for control group treatments. Platelet aggregation was induced using $10 \mu \mathrm{mol} / 1 \mathrm{ADP}, 10 \mu \mathrm{mol} / 1$ epinephrine, $1.0 \mathrm{mmol} / 1$ arachidonic acid (AA), $1.5 \mathrm{mg} / \mathrm{ml}$ ristocetin, $0.769 \mathrm{U} / \mathrm{ml}$ thrombin (Hunan Yige Pharmaceutical Co., Ltd., Dalian China), $1.304 \mathrm{U} / \mathrm{ml}$ oxytocin (Shanghai Harvest Pharmaceutical Co., Ltd., Shanghai, China) or $0.291 \mathrm{U} / \mathrm{ml}$ pituitrin (Anhui Hongye Pharmaceuticals Co., Ltd., Bengbu, China). Each agonist was applied alone, or in combination with synthetic polyphosphate (6 or $10 \mu \mathrm{l}$ ), and the aggregation rate was determined.

Platelet $\mathrm{Ca}^{2+}$ evaluation via flow cytometry. A total of $20 \mu \mathrm{l}$ PRP, treated using $10 \mu \mathrm{mol} / \mathrm{l} \mathrm{ADP}$ as described in the platelet aggregation assay, was diluted 1:10 in modified Tyrode's solution. Platelet $\mathrm{Ca}^{2+}$ concentrations were then determined as previously described (10), with the modification that the platelets were labeled with CD61-PerCP (BD Biosciences, Franklin Lakes, NJ, USA), not CD41. The CD61-PerCP-labeled platelets and Fluo3-chelated calcium concentrations in the platelets were detected using a BD FACSAria III cell sorter (BD Biosciences). All experiments were performed in triplicate.

Platelet $\mathrm{Ca}^{2+}$ evaluation via imaging. A total of $10 \mu \mathrm{l}$ PRP, treated with $10 \mu \mathrm{mol} / 1 \mathrm{ADP}$ as described above, was placed in 96-well plates (PerkinElmer, Inc., Waltham, MA, USA) and dyed with CD61-PerCP and Fluo-3 for 20 min prior to 1:10 dilution with modified Tyrode's buffer. The plate was centrifuged at 13,000 x g for 5 min (Centrifuge 5804R; Eppendorf, Hamburg, Germany) and immediately analyzed using the 40x long working distance objective of a high-throughput screening system (Operetta; PerkinElmer, Inc.). The system was equipped with a 460-490 nm excitation filter, a 500-530 nm long-pass (LP) emission filter for Fluo-3, and a 650-690 nm LP emission filter for CD61-PerCP. All experiments were performed in triplicate.

Thromboxane B2 (TXB2) measurement. To assess thromboxane A2 (TXA2) levels, the stable metabolite TXB2 was analyzed using a commercial ELISA kit (R\&D Systems, Inc., Minneapolis, MN, USA) (11). Briefly, PRP, treated with $10 \mu \mathrm{mol} / 1 \mathrm{ADP}$ as described above, was quenched for $5 \mathrm{~min}$ with $5 \mathrm{mM}$ ethylenediamine tetra-acetic acid and $200 \mu \mathrm{M}$ indomethacin to inhibit further TXA2 formation. The samples were centrifuged for $4 \mathrm{~min}$ at 12,000 $\mathrm{x} \mathrm{g}$. The supernatant was removed and stored at $-80^{\circ} \mathrm{C}$ for subsequent TXB2 analysis using an Infinite M200pro NanoQuant plate reader (Tecan, Männedorf, Switzerland).

Statistical analysis. The data were analyzed with SAS 8.1 (SAS Institute, Cary, NC, USA), and $\mathrm{P}<0.05$ was considered to indicate a statistically significant difference. The data were analyzed using nonparametric analysis of variance (ANOVA) based on the heterogeneity of variance, paired t-test or one-way ANOVA. 
Table I. Routine coagulation test results following the addition of synthetic polyphosphate (polyP).

\begin{tabular}{|c|c|c|c|c|c|c|}
\hline Parameters & Control & $5 \mu 1$ polyP & $10 \mu 1$ polyP & $20 \mu 1$ polyP & $40 \mu 1$ polyP & Reference range \\
\hline PT, sec & 11.72 & 12.40 & 12.10 & 13.20 & 13.90 & $10.0-16.0$ \\
\hline РТ\% & 122.73 & 114.77 & 119.32 & 105.68 & 97.73 & $70-140$ \\
\hline INR & 0.91 & 0.97 & 0.93 & 1.03 & 1.08 & $0.8-1.2$ \\
\hline APTT, sec & 37.30 & 50.90 & 60.50 & 83.63 & $>180.0$ & $25.0-43.0$ \\
\hline TT, sec & 19.50 & 19.60 & 18.9 & 24.5 & 26.9 & $14.0-21.0$ \\
\hline FIB, g/l & 2.17 & 3.19 & 3.41 & 2.01 & 1.98 & $2.0-4.0$ \\
\hline $\mathrm{DD}, \mathrm{mg} / \mathrm{l}$ & 0.48 & 0.46 & 0.16 & 0.15 & 0.07 & $0-0.5$ \\
\hline
\end{tabular}

PT, prothrombin time; INR, international normalized ratio; APTT, activated partial thromboplastin time; TT, thrombin time; FIB, fibrinogen; DD, D-dimer.

Table II. APTT test results at 0 and $4 \mathrm{~h}$ after the addition of synthetic polyphosphate (polyP).

\begin{tabular}{lccc}
\hline \multirow{2}{*}{ Group } & \multicolumn{3}{c}{ APTT $(\mathrm{sec})$} \\
\cline { 2 - 4 } & $0 \mathrm{~h}$ & $4 \mathrm{~h}$ & Reference range \\
\hline Control & 37.10 & 35.10 & $25.0-43.0$ \\
$5 \mu 1$ polyP & 50.90 & 37.30 & \\
$10 \mu 1$ polyP & 66.90 & 54.10 & \\
$20 \mu 1$ polyP & 80.10 & 63.00 & \\
\hline
\end{tabular}

APTT, activated partial thromboplastin time.

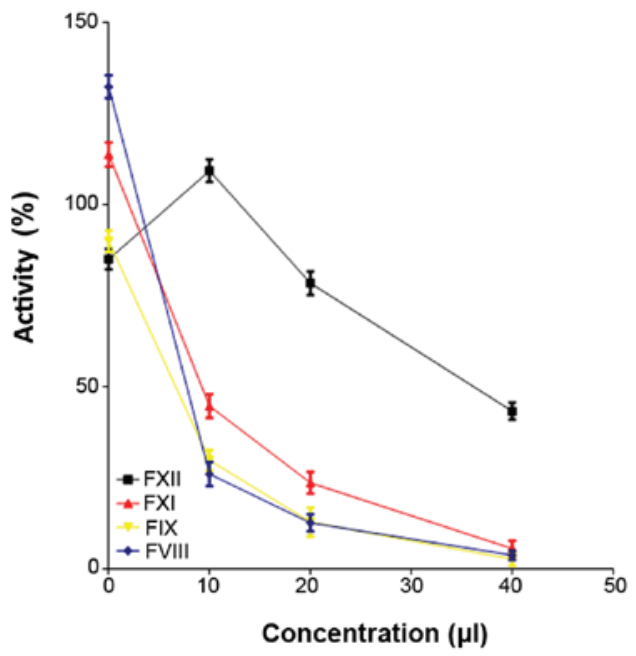

Figure 1. Synthetic polyphosphate inhibits the activity of intrinsic coagulation factors. The activities of coagulation factors FVIII, FIV and FXI were decreased by the addition of synthetic polyphosphate. The addition of $10 \mu 1$ polyphosphate increased FXII activity; however, the activity of FXII remained within the normal range. At higher polyphosphate levels, FXII activity declined as the concentration of polyphosphate increased. Overall, synthetic polyphosphate inhibited clotting factor activity.

\section{Results}

Synthetic polyphosphate inhibits coagulation. The addition of $250 \mathrm{mM} \mathrm{LiCl}$ did not impact routine coagulation
Table III. Activity of coagulation factors FXII, FXI, FVIII and FIV declined with increasing concentrations of synthetic polyphosphate (polyP).

\begin{tabular}{lcccc}
\hline Group & FVIII (\%) & FIX (\%) & FXI (\%) & FXII (\%) \\
\hline Control & 132.5 & 90 & 113.9 & 85.1 \\
$10 \mu 1$ polyP & 26 & 29.8 & 44.7 & 109.4 \\
$20 \mu 1$ polyP & 12.6 & 12.8 & 23.6 & 78.5 \\
$40 \mu 1$ polyP & 3.7 & 2.6 & 5.5 & 42.2 \\
Reference range & $60-150$ & $60-120$ & $70-120$ & $70-150$ \\
\hline
\end{tabular}

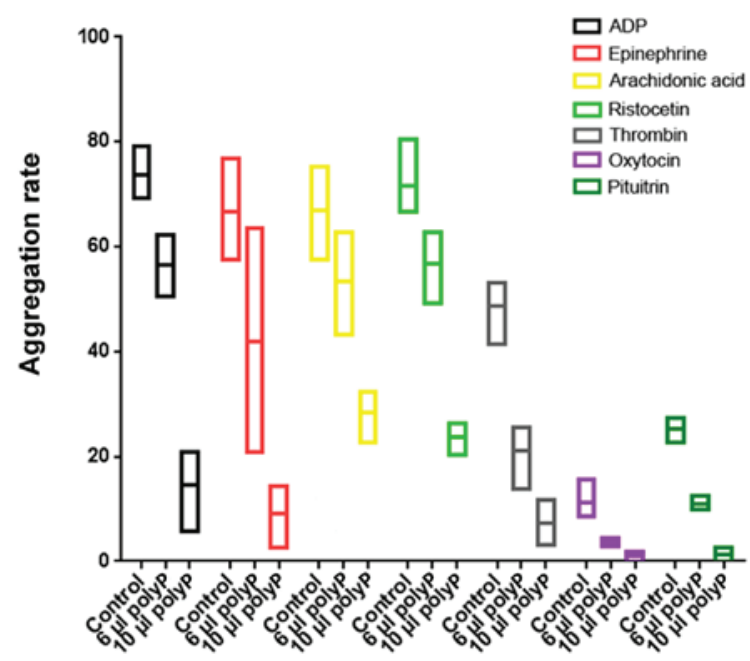

Figure 2. Maximal aggregation rate was inhibited by synthetic polyphosphate. Synthetic polyphosphate (polyP) inhibited the platelet maximal aggregation rate induced by adenosine diphosphate (ADP), platelet epinephrine, arachidonic acid, ristocetin, thrombin, oxytocin and pituitrin. As the polyP concentration was increased, the suppression was increased.

and was used as the control group treatment. Since FIB, DD and PT showed no significant changes from the control values when polyphosphate was added, this indicated that synthetic polyphosphate did not significantly affect the exogenous coagulation pathway and fibrinolysis. However, APTT, representing endogenous coagulation, increased significantly following the addition of synthetic polyphosphate $(\mathrm{F}=51.00$, 
Table IV. Thromboelastography results.

\begin{tabular}{|c|c|c|c|c|c|c|c|c|c|c|}
\hline Group & $\mathrm{R}(\min )$ & $\mathrm{K}(\min )$ & Angle degree & MA (mm) & $\mathrm{G}(\mathrm{K})$ & TMA (min) & CI & LTE (min) & $\operatorname{EPL}(\%)$ & LY30 (\%) \\
\hline Control & 5.8 & 1.7 & 63.4 & 64.7 & 9.2 & 27.1 & 0.5 & 32.8 & 0 & 0 \\
\hline $20 \mu 1$ polyP & 6.8 & 2.1 & 61.5 & 57.9 & 6.9 & 28.2 & -1.2 & 127.2 & 0.8 & 0.8 \\
\hline $40 \mu 1$ polyP & 9.8 & 2.6 & 54.5 & 58.6 & 7.1 & 32.1 & -3.4 & $>3 \mathrm{~h}$ & 0 & 0 \\
\hline $80 \mu 1$ polyP & 10.9 & 3.3 & 47.1 & 56.7 & 6.5 & 39.2 & -5.6 & $>3 \mathrm{~h}$ & No result & No result \\
\hline Reference range & $2-8$ & $1-3$ & $56-69$ & $51-69$ & 4.6-10.9 & - & -3 to +3 & - & $0-15$ & $0-8$ \\
\hline
\end{tabular}

$\mathrm{R}$, reaction time; $\mathrm{K}$, time required to reach $20 \mathrm{~mm}$ amplitude; MA, maximum amplitude; $\mathrm{G}$, shear elastic simulation strength; TMA, time to maximum amplitude; CI, coagulation index; LTE, lysis time estimate; EPL, estimated percent lysis; LY30, percentage of clot lysed after 30 min; polyP, synthetic polyphosphate.

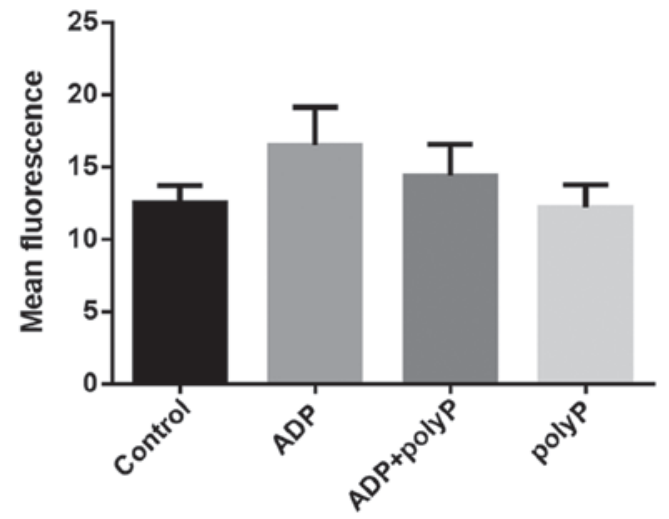

Figure 3. Calcium concentration was inhibited by synthetic polyphosphate, as detected by flow cytometry. The mean fluorescence intensity of platelet calcium in the adenosine diphosphate (ADP) group was higher than that in the control group $(16.49 \pm 2.65$ vs. $12.48 \pm 1.23)$. When $10 \mu 1$ synthetic polyphosphate (polyP) was added in combination with ADP, the mean calcium fluorescence intensity decreased to $14.39 \pm 2.18(\mathrm{P}<0.05)$. There was no significant difference between the control group and the polyP alone group $(12.48 \pm 1.23$ vs. $12.19 \pm 1.57)$.

$\mathrm{P}<0.0001$; Table I). As the half-life of synthetic polyphosphate is $\sim 2 \mathrm{~h}(6)$, the whole blood samples were allowed to stand for $4 \mathrm{~h}$ following the addition of 5, 10 or $20 \mu \mathrm{l}$ polyphosphate in $\mathrm{LiCl}$, or $\mathrm{LiCl}$ alone, prior to APTT testing. The APTT changed little over $4 \mathrm{~h}$ in the $\mathrm{LiCl}$ group, whereas the APTT was significantly increased in the synthetic polyphosphate groups $(\mathrm{F}=15.25, \mathrm{P}=0.0298$; Table II).

Synthetic polyphosphate inhibits intrinsic coagulation factor activity. The FVIII ( $\mathrm{F}=3908.37, \mathrm{P}=0.0001)$, FIX ( $\mathrm{F}=1590.24$, $\mathrm{P}<0.0001)$ and FXI $(\mathrm{F}=542.24, \mathrm{P}<0.0001)$ activities were decreased following the addition of polyphosphate (Fig. 1 and Table III), whereas $\mathrm{LiCl}$ (control) did not affect coagulation factor activity $(\mathrm{F}=50.02, \mathrm{P}=0.9971)$. A $10-\mu 1$ volume of synthetic polyphosphate increased FXII activity, but the FXII activity remained within the normal range. Furthermore, FXII activity declined as the concentration of polyphosphate increased $(\mathrm{F}=489.10, \mathrm{P}<0.0001)$. Overall, the synthetic polyphosphate inhibited clotting factor activity and blood coagulation, as assessed using conventional coagulation tests.

TEG confirms that synthetic polyphosphate inhibits blood clotting. $\mathrm{LiCl}$ did not affect TEG and was therefore used as a control treatment. As the concentration of synthetic polyphosphate increased, $\mathrm{R}(\mathrm{F}=3030.29, \mathrm{P}<0.0001)$ and TMA $(\mathrm{F}=3180.50, \mathrm{P}<0.0001)$ increased but the MA $(\mathrm{F}=115.78$, $\mathrm{P}<0.0001), \mathrm{G}$ value $(\mathrm{F}=159.55, \mathrm{P}<0.0001)$ and $\mathrm{CI}(\mathrm{F}=3581.71$, $\mathrm{P}<0.0001)$ decreased. These results indicate that polyphosphate inhibited coagulation. Thromboelastograms indicated that polyphosphate decreased significantly clotting factor activity and platelet function, since the angle degree decreased $(\mathrm{F}=455.28, \mathrm{P}<0.0001)$ and the $\mathrm{K}$ value increased $(\mathrm{F}=285.50$, $\mathrm{P}<0.0001)$. However, when $20 \mu$ l polyphosphate was added to $3 \mathrm{ml}$ whole blood, the APTT for routine coagulation was approximately twice the normal value, and was significantly abnormal $(\mathrm{P}=0.0026)$. Furthermore, at this time, the $\mathrm{R}$, TMA, MA, G value and CI obtained by TEG, representing the clotting factor activity or platelet function, and EPL and LY30, representing the fibrinolytic system, were in the normal range (Table IV). The LTE is an estimate of the clot lysis time. However, the results indicate that LTE more accurately predicts decreased clotting factor activity and platelet function. When $20 \mu \mathrm{l}$ polyphosphate was added to $3 \mathrm{ml}$ whole blood, the $\mathrm{R}$ and MA remained in the normal range, and at this time the LTE increased from 32.8 to $127.2 \mathrm{~min}$, which far exceeded the normal value. When $80 \mu \mathrm{l}$ polyphosphate was added to $3 \mathrm{ml}$ whole blood, TEG was not able to provide results for LTE, EPL and LY30; this may be due to synthetic polyphosphate severely inhibiting blood coagulation by depressing the activity of platelets and coagulation factors, so that neither blood coagulation nor fibrinolysis occurred. The fibrinolytic system might not necessarily be abnormal. Thus, the routine coagulation test is more accurate for the detection of coagulation and fibrinolysis than thromboelastograms.

Synthetic polyphosphate inhibits platelet aggregation. Synthetic polyphosphate inhibited ADP (F=72.51, $\mathrm{P}<0.0001)$-, platelet epinephrine $(\mathrm{F}=13.74, \mathrm{P}=0.0058)-$, AA $(\mathrm{F}=19.19$, $\mathrm{P}=0.0025)-$, ristocetin $(\mathrm{F}=66.16, \mathrm{P}<0.0001)-$, thrombin $(\mathrm{F}=41.59, \mathrm{P}=0.0003)$-, oxytocin $(\mathrm{F}=30.61, \mathrm{P}=0.0007)-$ and pituitrin $(\mathrm{F}=202.14, \mathrm{P}<0.0001)$-induced platelet aggregation. As the concentration of polyphosphate increased, suppression was increased (Fig. 2).

Synthetic polyphosphate reduces calcium concentrations with platelet aggregation. The mean fluorescence intensity of platelet calcium in the ADP group was higher than that in the 


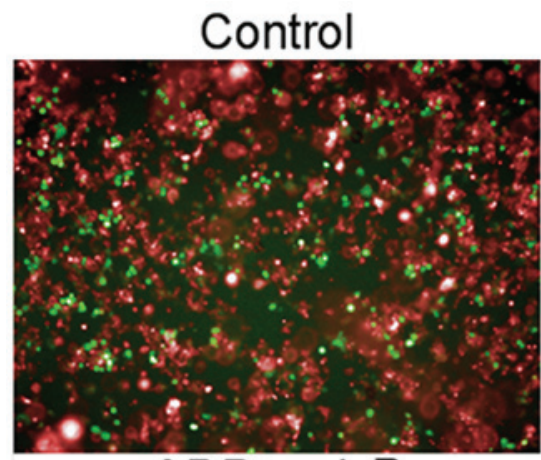

ADP+polyP

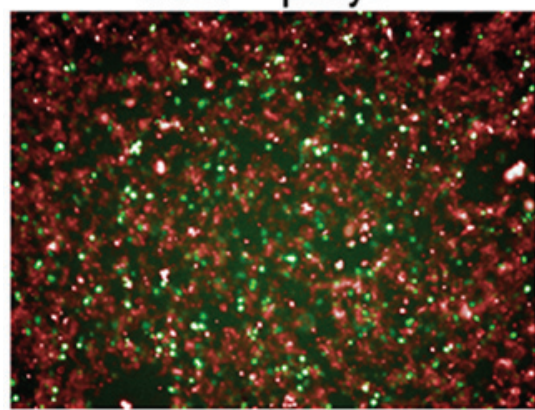

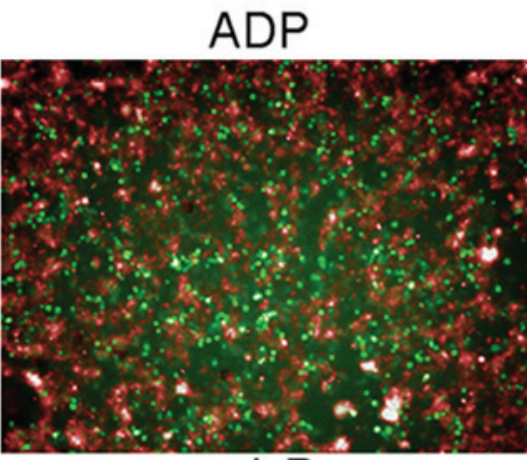

polyP

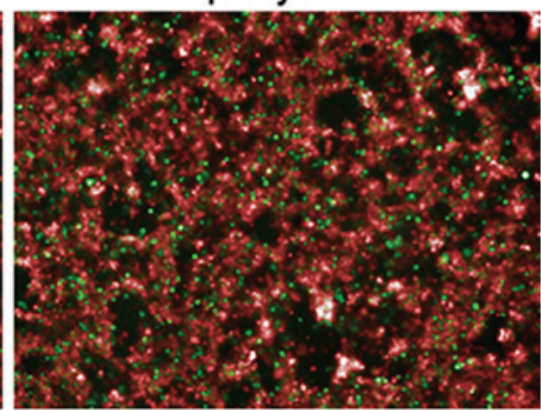

Figure 4. Synthetic polyphosphate inhibits calcium concentrations along with platelet aggregation. Fluorescence imaging of platelets (magnification, x400). The red fluorescence indicates platelets dyed with CD61-PerCP, and the green fluorescence indicates calcium dyed with Fluo-3. Calcium levels in platelets to which adenosine diphosphate (ADP) was added were higher than those in the control group. When $10 \mu 1$ synthetic polyphosphate (polyP) was administered in combination with ADP, the calcium level was decreased compared with that in the ADP group. There was no obvious difference between the control group and platelets treated with polyP alone.

control group $(16.49 \pm 2.65$ vs. $12.48 \pm 1.23 ; \mathrm{P}=0.0135)$. When $10 \mu \mathrm{l}$ synthetic polyphosphate and ADP were both applied, the mean calcium fluorescence intensity decreased to $14.39 \pm 2.18$ $(\mathrm{P}=0.0031)$; however, there was no significant difference between the control group and the polyphosphate group $(12.48 \pm 1.23$ vs. $12.19 \pm 1.57 ; \mathrm{P}=0.4462 ;$ Fig. 3$)$. The same pattern of results was obtained via high-throughput imaging (Fig. 4). Thus, it appears that synthetic polyphosphate inhibits platelet aggregation and activation by reducing calcium levels.

Synthetic polyphosphate inhibits TXA2. Treatment of platelets with polyphosphate significantly decreased TXB2 levels compared with those in untreated platelets $(1.416 \pm 0.248$ vs. $0.409 \pm 0.464 ; \mathrm{P}<0.0001)$. By contrast, ADP increased TXB2 levels, and the ADP-induced increases were attenuated by polyphosphate $(1.701 \pm 0.367$ vs. $1.146 \pm 0.235 ; \mathrm{P}<0.0001)$.

\section{Discussion}

Previous studies have revealed that platelet-derived polyphosphate promotes blood coagulation $(5,6)$. However, the effects obtained when adding synthetic polyphosphate have been conflicting. In the present study, endogenous clotting factor (i.e., FXII, FVIII, FIV and FXI) activity was inhibited following the addition of synthetic polyphosphate. APTT, which represents the endogenous coagulation time and is used to guide clinical treatment, was prolonged and higher than the diagnostic criteria of DIC (12). The effects on fibrinogen and DD were small, with the results varying within the normal range. The changes in routine coagulation caused by synthetic polyphosphate gradually weakened as the polyphosphate underwent degradation for $4 \mathrm{~h}$, as assessed in the routine coagulation test of the present study $(\mathrm{F}=15.25, \mathrm{P}=0.0298)$. Synthetic polyphosphate turnover is dynamic; after the polyphosphate enters the plasma, changes in its integrity, size and concentration occur (13). In addition, its half-life in plasma is 1.5-2 $\mathrm{h}$ (14). Therefore, it is difficult to accurately measure the concentration of synthetic polyphosphate with linked phosphate groups (13).

LTE, an estimate of clot lysis time, may predict platelet function more accurately than fibrinolysis, as determined by comparing thromboelastograms, conventional coagulation times and reports from the literature. Thromboelastograms may not be accurate for predicting fibrinolysis. Routine coagulation tests exhibit difficulty in detecting the effects of higher concentrations of synthetic polyphosphate, and thromboelastograms exclusively detect abnormalities. However, at lower concentrations, routine coagulation tests are more accurate than thromboelastograms. Therefore, TEG may accurately predict severe, life-threatening coagulopathy, while routine coagulation tests perform better than TEG when predicting small changes in coagulopathy (15-17).

Synthetic polyphosphate and platelet-derived polyphosphate have different effects on coagulation. One possible reason for this is that these two phosphates are have different chemical structures. The polyphosphate anion is able to bind calcium ions (2). The mechanism of the reaction between calcium ions and sodium polyphosphate is not thoroughly understood but is considered to be as follows:

$$
\begin{aligned}
& \left(\mathrm{NaPO}_{3}\right)_{\mathrm{n}} \rightarrow 2 \mathrm{Na}^{+}+\mathrm{Na}_{\mathrm{n}-2}\left(\mathrm{PO}_{3}\right)^{2-} \\
& \mathrm{xCa}^{2+}+\mathrm{Na}_{\mathrm{n}-2}\left(\mathrm{PO}_{3}\right)_{\mathrm{n}}^{2-} \rightarrow 2 \mathrm{xNa}^{+}+\mathrm{Na}_{\mathrm{n}-(2+2 \mathrm{x})} \mathrm{Ca}_{\mathrm{x}}\left(\mathrm{PO}_{3}\right)_{\mathrm{n}}^{2-}
\end{aligned}
$$


The synthetic polyphosphate used in the present study, and polyphosphate derived from cells have different structures, which may affect their functions. The polyphosphate in platelets or cells is calcium-saturated polyphosphate (calcium polyphosphate). The synthetic polyphosphate used in the present study was sodium polyphosphate. Sodium polyphosphate can bind to calcium in plasma and inhibit coagulation and the activity of coagulation factors. Calcium polyphosphate cannot bind calcium ions in plasma, and accelerates coagulation by activating factor $\mathrm{V}$ or factor XI (5).

Platelet aggregation tests in plasma are calcium-free; therefore synthetic polyphosphate could not bind calcium in the plasma. Therefore, the mechanisms by which synthetic sodium polyphosphate inhibited platelet aggregation were investigated in the present study, and it was found that the synthetic polyphosphate inhibited both calcium and TXA2.

TXA2 is produced by activated platelets and has prothrombotic properties. It also stimulates the activation of new platelets and increases platelet aggregation. TXA2 is unstable in aqueous solution and is hydrolyzed within $\sim 30 \mathrm{sec}$ to the biologically inactive TXB2 (18). In human studies, TXB2 levels are used to indirectly measure TXA2 production (19). When clotting begins in the body, calcium mobilization occurs in the dense tubular system. Increased intracellular calcium is associated with the activation of several kinases that are necessary for morphological changes, presentation of the procoagulant surface, secretion of platelet granules, activation of glycoproteins, and activation of phospholipase A2 (PLA2). PLA2 activation releases AA, a TBXA2 precursor, with prostaglandin $\mathrm{G} / \mathrm{H}$ synthase 1 catalyzing the first step in the formation of TBXA2 from AA. These processes result in the local accumulation of molecules such as thrombin, TXA2 and ADP, which are important for further platelet aggregation (20). The results of the TXA2 assay conducted in the present study indicate that this reaction was blocked by synthetic polyphosphate, thereby reducing platelet aggregation.

In conclusion, synthetic polyphosphate inhibits endogenous coagulation and platelet aggregation in vitro. The effects of synthetic polyphosphate on coagulation are different from those of platelet-derived polyphosphate. Synthetic polyphosphate may be a potential drug for the prevention or treatment of thrombosis. Obviously, whether the complex compound binds with calcium or not, or changes in concentration, may induce different effects or cause damage to the body. Further investigation of the role of synthetic polyphosphate in the body is necessary in future studies.

\section{Acknowledgements}

The authors would like to express their warmest gratitude to all of the nurses of Xiangya Hospital and Xiangya Third Hospital of Central South University as well as all their colleagues in the clinical testing laboratory of Xiangya Hospital and Xiangya Third Hospital of Central South University.

\section{References}

1. Brown MR and Kornberg A: Inorganic polyphosphate in the origin and survival of species. Proc Natl Acad Sci USA 101: 16085-16087, 2004.

2. Schwartz C, Jones KK, Mack TW and Vance RW: The use of sodium metaphosphate for the preparation of soft-curd milk. J Dairy Sci 23: 19-35, 1940.

3. Wiame JM: Yeast metaphosphate. Fed Proc 6: 302, 1947.

4. Jimenez-Nuñez MD, Moreno-Sanchez D, Hernandez-Ruiz L, Benítez-Rondán A, Ramos-Amaya A, Rodríguez-Bayona B, Medina F, Brieva JA and Ruiz FA: Myeloma cells contain high levels of inorganic polyphosphate which is associated with nucleolar transcription. Haematologica 97: 1264-1271, 2012.

5. Faxälv L, Boknäs N, Ström JO, Tengvall P, Theodorsson E, Ramström S and Lindahl TL: Putting polyphosphates to the test: evidence against platelet-induced activation of factor XII. Blood 122: 3818-3824, 2013.

6. Smith SA, Choi SH, Davis-Harrison R, Huyck J, Boettcher J, Rienstra CM and Morrissey JH: Polyphosphate exerts differential effects on blood clotting, depending on polymer size. Blood 116: 4353-4359, 2010.

7. Yang X, Wan M, Yang K and Chen F: Long chain polyphosphates identified in infectious fever patients in the department of hematology. Acta Med Mediterr 32: 377-383, 2016.

8. Levi M, Toh CH, Thachil J and Watson HG; British Committee for Standards in Haematology: Guidelines for the diagnosis and management of disseminated intravascular coagulation. Br J Haematol 145: 24-33, 2009.

9. Kol A and Borjesson DL: Application of thrombelastography/thromboelastometry to veterinary medicine. Vet Clin Pathol 39: 405-416, 2010.

10. do Céu Monteiro M, Sansonetty F, Gonçalves MJ and O'Connor JE: Flow cytometric kinetic assay of calcium mobilization in whole blood platelets using Fluo-3 and CD41. Cytometry 35: 302-310, 1999.

11. Walsh TG, Harper MT and Poole AW: SDF-1 $\alpha$ is a novel autocrine activator of platelets operating through its receptor CXCR4. Cell Signal 27: 37-46, 2015.

12. Wada H, Matsumoto T and Yamashita Y: Diagnosis and treatment of disseminated intravascular coagulation (DIC) according to four DIC guidelines. J Intensive Care 2: 15, 2014.

13. Lorenz B and Schröder HC: Methods for investigation of inorganic polyphosphates and polyphosphate-metabolizing enzymes. Prog Mol Subcell Biol 23: 217-239, 1999.

14. Smith SA, Mutch NJ, Baskar D, Rohloff P, Docampo R and Morrissey JH: Polyphosphate modulates blood coagulation and fibrinolysis. Proc Natl Acad Sci USA 103: 903-908, 2006.

15. Katz-Summercorn AC, Cuffolo G, Hossain MA and Wilde M: The use of rapid thromboelastogram for trauma mortality prediction. Am J Surg 208: 316, 2014.

16. Tanriverdi S, Koroglu OA, Uygur O, Balkan C, Yalaz M and Kultursay N: The effect of inhaled nitric oxide therapy on thromboelastogram in newborns with persistent pulmonary hypertension. Eur J Pediatr 173: 1381-1385, 2014.

17. Valeri CR and Ragno G: In vitro testing of platelets using the thromboelastogram, platelet function analyzer, and the clot signature analyzer to predict the bleeding time. Transfus Apheresis Sci 35: 33-41, 2006.

18. Fontana P, Zufferey A, Daali Y and Reny JL: Antiplatelet therapy: targeting the TxA2 pathway. J Cardiovasc Transl Res 7: 29-38, 2014.

19. Lordkipanidzé M, Pharand C, Schampaert E, Turgeon J, Palisaitis DA and Diodati JG: A comparison of six major platelet function tests to determine the prevalence of aspirin resistance in patients with stable coronary artery disease. Eur Heart J 28: 1702-1708, 2007.

20. Sangkuhl K, Shuldiner AR, Klein TE and Altman RB: Platelet aggregation pathway. Pharmacogenet Genomics 21: 516-521, 2011. 